\title{
The effects of extraneous response requirements on learning by performers and observers
}

\author{
MILTON E. ROSENBAUM AND LINDSLEY J. SCHUTZ \\ UNIVERSITY OF IOWA
}

On a first trial, Ss explored a multiple-choice maze. Performers (Ps), in the presence of observers (Os), utilized either a stylus or a radio tube to make each response. The decision as to which response to make was made either by Ps themselves or by Os who told Ps which responses to make. Decision making and complex response requirements (Tube manipulation) were found to interfere with performance on a retention test.

In many tasks, in addition to carrying out the activities directly relevant to successful criterion performance, other nonrelevant activities are frequently demanded of a performer. For example, Hillix \& Marx (1960) suggested that in a multiple-choice task, the necessary but nonfunctional activity of deciding among responses may interfere with optimal learning of correct choice location.

In any response chain, a variety of such nonfunctional activities may of necessity occur. In addition to deciding among responses, the amount of perceptual-motor activity necessary for a performer to complete a response may be exaggerated and unnecessary for successful criterion performance and hence interfere with optimal learning.

In the present experiment a comparison of the retention of correct responses by performers and observers in a multiple-choice maze situation was examined. Also examined were two additional experimental variables: the role of decision making and the role of increased perceptual-motor activity. During an initial exploratory trial, decision making responsibility was assigned either to a performer or an observer. The implement which the performer used to make his response was varied by having him employ either a stylus that required minimal perceptual-motor activity or a radio tube that demanded considerable manipulative and/or perceptual-motor activity.

It was hypothesized that both decision making and increased perceptual-motor activity (tube condition) would interfere with learning.

\section{Apparatus}

The apparatus was a multiple-choice maze consisting of 80 eight-pin radio tube sockets in a 4 by 20 matrix, mounted on an aluminum panel $1 \times 4 \mathrm{ft}$. in size. Sockets were placed $1 \mathrm{in}$. apart and the keyway of each socket was directionally random. Six equidistant screws surrounded each socket to eliminate cues to direction of keying. The panel was placed on a table, and also on the table, back of the panel, was a box with a red and a green jeweled lamp. The green light was designated as signaling a correct response, and one socket in each of the 20 rows of four sockets was wired to this light. The other three sockets in each row were wired to the red light which indicated an incorrect response. The socket wired to the green light was selected randomly with sole restriction that no more than two sockets in the same column could occur in no more than two successive rows. The four columns of sockets were labeled with numbers 1 to 4 . Ss made a response by placing a stylus made from a ball-point pen through the hole in the center of a socket which made contact with bolt-heads under the panel or Ss responded by placing a 6AG7 radio tube in the socket. The stylus was inserted readily while the tube had to be lined up with the socket keyway.

\section{Procedure}

Performers (Ps) were instructed to insert the prescribed implement into the tube sockets to locate the socket in each row thatilluminated the green light. Each response was to be made following a buzzer signal which sounded every $10 \mathrm{sec}$. Exploration of the maze was to proceed row by row from the top of the maze with the restriction that the implement must be inserted into the correct socket in each row before proceeding to the next row. P stood so that the right side of his body was perpendicular to the long side of the maze panel and he employed his right hand in implement manipulation. In each experimental session an observer (O) stood near P's left side facing the maze panel.

In a Performer-Decision condition (P-D), Ss were told that $P$ was to decide into which socket in each row he was going to insert the implement and call out his decision before performing the response by designating the column by number. In the Observer-Decision condition $(O-D), O$ chose and called out the column number into which $\mathbf{P}$ inserted the implement. In each of these conditions, the $S$ not instructed to choose the response remained silent creating two additional conditions, Performer-No Decision (P-ND) and Observer-No Decision (O-ND). Half of the Ps in each of the above conditions used a Tube (Tu) as the implement and half employed the Stylus (St). Both Ss were informed that their retention of the maze pattern would be tested independently after the exploratory trial.

After completing the last row, $P$ and $O$ were placed apart in the room and given the retention test. The test consisted of a dittoed sheet on which the maze was represented by a matrix of 4 by 20 circles. Ss were instructed to indicate the correct circle (socket) in each row. Ss completed the test at their own pace.

The Ss who appeared in pairs were 112 male introductory psychology students. They were randomly assigned in equal numbers to the eight conditions repre- 
Table 1. Mean Number of Responses on Exploratory Trial and Mean Number Correct Responses on Retention Test

\begin{tabular}{lccc} 
Condition & $\begin{array}{c}\text { Exploratory Trial } \\
\text { No. of Responses }\end{array}$ & $\begin{array}{c}\text { Retention Test } \\
\text { No. Correct } \\
\text { Observer }\end{array}$ \\
\hline $\begin{array}{l}\text { Stylus } \\
\text { Decision }\end{array}$ & 46.50 & $9.64^{a}$ & $8.64^{a}$ \\
$\quad$ No Decision & 48.71 & $12.36^{b}$ & $9.50^{a}$ \\
Tube & & & \\
Decision & 46.93 & $8.29^{a}$ & $7.64^{a}$ \\
No Decision & 46.14 & $8.79^{a}$ & $12.64^{b}$ \\
\hline
\end{tabular}

sented by two levels of Participation ( $P$ and $O$ ), two levels of Decision (D and ND), and two kinds of Implements (Tu and $\mathrm{St}$ ).

Results and Discussion

Table 1 presents the mean number of responses made on the first (exploratory) trial for each group. A 2 by 2 analysis of variance indicated no significant effects.

The mean number of correct responses by each group on the retention task appears also in Table 1 . A main effect for Decision $(F=11.80, \mathrm{df}=1 / 104, p<.01)$ is indicative of superior retention in the ND conditions as compared with the $\mathrm{D}$ conditions. The Implement by Participation interaction $(F=7.17, \mathrm{df}=1 / 104, \mathrm{p}<.01)$ was significant. A critical difference (Lindquist, 1953) between condition means of 1.89 is required for significance at the .05 level. Retention in the P-St conditions (mean $=11.00$ ) is superior to that in the P-Tu conditions (mean $=8.54$ ), while no such differences occur between the O-St (mean=9.07) and O-Tu (mean=10.14) conditions. This finding suggests that the tube manipulation interfered with learning as hypothesized. Also, the retention of Ss in the P-St conditions is superior to that of $\mathrm{Ss}$ in the O-St conditions while no such differences occur between Ps and Os in the Tu conditions. A significant Implement by Participation by Decision interaction ( $F=5.80$, df $=1 / 104, p<.05$ ) was also found. The means found not to be significantly different from each other are marked by common letters in Table 1. A difference of 2.59 is required for significance at the .05 level.

These comparisons reveal that Ps who carry out the more simple St task and are not required to decide among the choices show superior performance. Os who

Table 2. Mean Number Correct Responses on Retention Test in Preliminary Experiment

\begin{tabular}{lcr} 
Condition & Performer & Observer \\
\hline Stylus & 10.73 & 12.87 \\
Tube & 8.60 & 13.27 \\
\hline
\end{tabular}

observe the more complex Tu task and do not decide among the choices also manifest superior performance. It may be suggested then that performing irrelevant responses such as those required in the $\mathrm{P}-\mathrm{Tu}$ condition independent of the decision requirement interferes with acquisition. It also appears that decision making is generally deleterious to acquisition. The general conclusion may be offered that task activities not directly relevant to criterion performance requirements interfere with the learning process.

The one result that is inconsistent with this conclusion appears for the O-St-ND condition in that these Ss did not participate in decision making. Since neither of the suggested interfering sources occur in this condition, superior performance should have appeared.

In a preliminary study employing the same apparatus, the P-O conditions and St-Tu conditions were varied much as in the present experiment with only three procedural differences. Ps always made the choice, Ss did not verbalize, and a confederate who replicated the Trial 1 performance of a previous $P$ responded in the presence of an $O$. The results of the preliminary experiment are presented in Table 2. The only significant finding was the superiority of Os over Ps.

In the present experiment Os in the St condition who did not decide the responses (ND) performed on the retention test at a level apparently inferior to their counterparts in the preliminary experiment (mean = 9.50 vs. mean $=12.87$ ). What may be an essential difference between the two experiments is that in the preliminary study, a confederate served to provide Os with the first trial responses of Ps who had performed earlier while in the present study naive Ss served as $\mathrm{Ps}$ in the presence of Os. Manipulation of the stylus is a clearly simple and rapid activity. A confederate may serve as a much more clear demonstrator than a naive $S$ who may obscure his responses by rapid activity, while a naive $S$, when employing the tube, could hardly fail to be as deliberate and clear as a confederate demonstrator.

The possibility that the number of responses made on the exploratory trial may be related to retention test performance was examined. Correlation coefficients ranged from 0.57 to -.02 for the eight conditions with only the correlation for the P-Tu-D condition significant $(p<.05)$. The overall correlation was .09 which is not significant.

\section{Reference}

Hillix, W. A., \& Marx, M. H. Response strengthening by information and effect on human learning. J. exp. Psychol., 1960, 60, 97-102.

\section{Note}

1. This study was supported by a grant (GS-228) to the senior author from the National Science Foundation. 